Their main inadequacy was that they had to be given at repeated intervals to a large proportion of the widely dispersed rural population provided with rudimentary health services. Furthermore, no antimalarial drug is equally suitable for prevention and treatment, for prescription to individual patients, and for large-scale distribution.

Nevertheless the range of known drugs with various specific actions appeared to be adequate. ${ }^{1}$ For treatment of acute malaria we had quinine, chloroquine, and amodiaquine apart from the obsolete mepacrine. For prevention of malaria there was a choice of proguanil, pyrimethamine, and chloroquine as a suppressant. For radical cure of relapsing malaria the standard treatment by 4-aminoquinolines (chloroquine, amodiaquine, and others) could be topped by primaquine. Attempts to improve the short action of proguanil and pyrimethamine by producing cycloguanil pamoate, an injectable derivative of the former drug, were encouraging.

The general complacency received the first jolt in the 1950s by the reports of resistance of malaria parasites to proguanil and pyrimethamine. Ten years later came another more serious shock owing to the apparent failure of chloroquine to cure some Plasmodium falciparum infections in Colombia, Brazil, Venezuela, and several countries of South-east Asia. Reports of resistance to chloroquine in other parts of the world and especially in Africa were not confirmed and proved to be unreliable. At the present time the main foci of resistance of $P$. falciparum to 4-aminoquinolines are still in the northwest of South America and in South-east Asia, but the possibility of its extension causes concern. ${ }^{2}$

The evidence of resistance of $P$. falciparum to chloroquine showed the relative poverty of our therapeutic measures and the narrowing margin of success in our fight against malaria. This became particularly obvious in the war areas of Southeast Asia, where the incidence of malaria in the military units increased strikingly since 1965, with frequent recrudescences of infection after standard treatment. In the meantime a partial answer to the problem of resistance of $P$. falciparum to 4aminoquinolines was found in the rediscovery of sulphones and sulphonamides as antimalarial drugs when combined with pyrimethamine or similar compounds.

For the past decade a large number of industrial laboratories, academic institutions, and independent scientists have been involved in a large programme which studied and screened over 150,000 compounds. The final results of this impressive collaborative endeavour are not yet available, as the clinical trials are not yet completed. Nevertheless, it appears that the drug screening programme carried out for the past ten years under the auspices of the U.S. Army has now produced several promising leads. Much of this success is due to the introduction of the experimental method based on the use of the Aotus or owl monkey, which can be infected with human plasmodia.

The results of these studies were presented recently in London to the Royal Society of Tropical Medicine and Hygiene. ${ }^{3}$ Four groups of compounds have been assessed: 9-phenanthrene methanols, 4-quinoline methanols, diaminoquinazolines, and diaminohydrotriazines. Several compounds among the first two groups have shown high activity on human volunteers.

The World Health Organization has tackled the prevention and treatment of malaria since its early days, but particularly during the past decade. ${ }^{45}$ The latest meeting of a W.H.O. scientific group on chemotherapy of malaria and resistance to antimalarial drugs took place in 1972. The report on this meeting, just published, ${ }^{6}$ reviews lucidly the use of these drugs in clinical practice and in malaria control or eradication programmes. It gives an outline of various aspects of resistance of human malaria to drugs and discusses the classification of the response of $P$. falciparum infections to chloroquine and of testing methods. It also describes the action of old and new antimalarial drugs and drug associations and provides some new information on the biochemical processes that regulate the growth of plasmodia and interfere with it. The report does not attempt to give an answer to many still unknown problems in this field but suggests some areas of promising research.

When it comes to a full understanding of the metabolism of malaria parasites or the mechanism of specific resistance we have not progressed very far from the 300-years-old statement of Sebastiano Badi that "the quotidian, tertian and quartan fevers are eliminated by the occult quality of the Cinchona bark... which must be included among the mirabilia...because it destroys the febrile fermentations".

\footnotetext{
1 Bruce-Chwatt, L. J., American fournal of Tropical Medicine and Hygiene, $1972,21,731$

Peters, W., Chemotherapy and Drug resistance in Malaria. New York, Academic Press, 1970.

Schmidt, L. H., Transactions of the Royal Society of Tropical Medicine and Hygiene, 1973, 67, 446.

World Health Organization, Technical Report Series No. 296. Geneva, W.H.O. 1965.

${ }^{5}$ World Health Organization, Technical Report Series No. 375. Geneva, W.H.O. 1967.

World Health Organization, Technical Report Series No. 529. Geneva, W.H.O. 1973 .
}

\section{Immunosuppressive Therapy in Active Chronic Hepatitis}

Before considering the use of immunosuppressive agents in the treatment of active chronic hepatitis it is necessary to examine the evidence that this is an autoimmune disease. The circulating autoantibodies so frequently present in the serum are not organ-specific but are capable of reacting with nuclei, mitochondria, or smooth muscle components in almost any organ. It is therefore unlikely that they have a direct role in the pathogenesis of a disease which is largely confined to the liver.

But recently liver-specific immunological reactions have been detected which could be more important in the production of liver damage. Meyer zum Buschenfelde, working in Mainz, has detected liver-specific autoantibodies in $10 \%$ of his patients with active chronic hepatitis and was able to isolate from human liver the liver-specific antigen against which these antibodies were directed. ${ }^{2}$

Evidence of hypersensitivity to antigens in liver tissue has been found in about half of a series of patients with active chronic hepatitis. Again, ${ }^{3}$ as with humoral antibodies, this sensitization would be more relevant to the pathogenesis if it were liver-specific. This has now been shown by the demonstration of cell-mediated immunity to one of the liver-specific antigens (referred to above) in $60 \%$ of patients with active chronic hepatitis. ${ }^{4}$

Both humoral and cellular mechanisms may co-operate to cause tissue damage. ${ }^{56}$ For immunosuppressive therapy to be effective it may therefore be necessary to suppress both parts of the immune response.

Corticosteroids have been used to treat active chronic hepatitis since the 1950 s and the first use of 6-mercaptopurine was reported in 1960. Since then some poorly controlled observations on treatment with these agents, as well as with azathioprine and chlorambucil, have suggested they caused 
clinical and biochemical improvement, but since the disease can have an unpredictable clinical course a prospective randomized trial was needed to test them.

The results of a controlled prospective trial carried out at the Royal Free Hospital were published in 1971. ${ }^{7}$ Prednisolone was compared with a placebo. The initial dose of prednisolone was $15 \mathrm{mg}$ daily, but this was reduced when the disease was controlled. After the trial had been in progress for six years 3 of the 22 corticosteroid-treated patients and 15 of the control patients had died, a highly significant difference in mortality. In a controlled trial carried out at King's College Hospital prednisone $15 \mathrm{mg}$ daily was"compared with azathioprine $75 \mathrm{mg}$ daily. At the end of two years' treatment the group on prednisone was found to be superior to the group on azathioprine in three respects. ${ }^{8}$ The fall of gammaglobulin was greater on prednisone, though the liver function tests improved in both groups. Oesophageal varices had developed in fewer patients on prednisone than on azathioprine, which suggests that only with the former drug was the progress of the disease checked. Finally, the calculated probability of surviving two years was $95 \%$ on prednisone and $72 \%$ on azathioprine.

However, the prednisone caused serious side effects, including crush fracture of vertebrae, diabetes mellitus, and systemic hypertension. This raises the question whether a smaller dose of prednisone alone or combined with azathioprine might be sufficient to control the disease. R. D. Soloway and colleagues, ${ }^{9}$ of the Mayo Clinic, showed that prednisone $20 \mathrm{mg}$ or a combination of prednisone $10 \mathrm{mg}$ and azathioprine $50 \mathrm{mg}$ was superior to azathioprine alone $(100 \mathrm{mg})$ or placebo in prolonging the life expectation and improving the biochemical and histological abnormalities. Serious side effects occurred in $21 \%$ of patients treated for more than 12 months with $20 \mathrm{mg}$ prednisone. These seemed to be less of a problem with the combination therapy.

The reasons for the high incidence of serious side effects from corticosteroid treatment have recently been clarified. At pharmacological doses many steroids, including cortisol (hydrocortisone) and prednisolone (the active metabolite of prednisone), are bound to serum albumin, and the lower the serum albumin the higher the percentage of unbound, biologically active steroid. In a survey of 240 medical inpatients receiving prednisone the frequency of side effects from it was found to be doubled when the serum albumin was less than $2.5 \mathrm{~g} / 100 \mathrm{ml} .^{10}$ With liver dysfunction the metabolism of prednisolone is impaired, ${ }^{11}$ and this could result in high blood levels of the drug for a long time.

Toxic effects on the liver of 6-mercaptopurine ${ }^{13}$ and azathioprine ${ }^{14}$ may present a problem. In patients with active chronic hepatitis they may be impossible to distinguish from the deterioration in liver function due to progression of the disease. Azathioprine hepatotoxicity, however, is unlikely if doses are kept to $75 \mathrm{mg}$ a day or less.

The aim of treatment is to give the smallest dose of prednisolone that will control the disease. This is usually about $15 \mathrm{mg} /$ day, though some patients are controlled only by maintenance doses of $20-30 \mathrm{mg} /$ day. If side effects become troublesome it may be possible to reduce the dose of prednisolone further by adding a small dose of azathioprine (50-75 $\mathrm{mg}$ /day). It seems that most of these patients will require treatment for at least some years. The experience of most centres is that even after two to three years' treatment many patients will relapse sooner or later after corticosteroids are withdrawn. But if liver function tests return to normal, and if a liver biopsy shows no evidence of continuing activity, then it is reasonable to try the effect of withdrawing treatment.

Another effective immunosuppressive agent-namely cyclophosphamide-has been used in occasional cases of active chronic hepatitis. It is more toxic than the drugs mentioned above for it may produce haemorrhagic cystitis as well as alopecia. Until a controlled assessment of its value has been made, it should not be used.

1 Meyer zum Buschenfelde, K. H., and Kossling, F. K., in Immunology of the Liver, p. 169, ed. M. Smith and R. Williams. London, Heinemann, 1971

2 Hopf, U., Meyer zum Buschenfelde, K. H., and Freudenberg, J., Clinical and Experimental Immunology, 1973, in press.

3 Smith, M. G. M., et al., British Medical fournal, 1972, 1, 527.

4 Miller, J., et al., Lancet, 1972, 2, 296.

Brown, P. C., Glynn, L. E., and Holborow, E. J., Immunology, 1967, 13, 307.

6 MacLennan, I. C. M., Loewi, G., and Howard, A., Immunology, 1969, 17,

7 Cook, G. C., Nulligan, R., and Sherlock, S., Quarterly fournal of Medicine, $1971,40,159$

${ }^{8}$ Murray-Lyon, I. M., Stern, R. B., and Williams, R., Lancet, 1973, 1, 735.

9 Soloway, R. D., et al., Gastroenterology, 1972, 63, 820.

10 Lewis, G. P., Jusko, W. J., Burke, C. W., and Graves, L., Lancet, 1971, 2, 778 .

11 Powell, L. W., and Axelsen, E., Gut, 1972, 13, 690.

12 Elion, G. B., Proceedings of the Royal Societv of Medicine, 1972, 65, 257.

13 Mistilis, S. P., and Blackburn, C. R. B., Australasian Annals of Medicine, $1967,16,305$.

14 Starzl, T. E., et al., Lancet, 1971, 2, 70.

\section{Sir George Godber Retires}

With the retirement at the end of November of Sir George Godber, Chief Medical Officer, from the Department of Health and Social Security the Department loses a chief who gave it a new sense of purpose. But, perhaps more important, the medical profession loses the services of a doctor who has done as much as anyone to advance medicine itself in this country.

When he was appointed C.M.O. in 1960 Sir George had already shown during the 20 years he had worked in the Ministry of Health a capacity to master the job that had made him familiar with much more than the mere details of the Health Service. He took the trouble to understand the problems that confront doctors daily in their practice, whether in the home, the hospital, the laboratory, or the local health offices and clinics. To an enviable capacity for work he added a patience and courtesy that as an official endeared him to a profession not notably tolerant of bureaucracy. And it is no secret that his knowledge and fair judgement have brought him an influence on successive Ministers of Health that must be exceedingly rare. The National Health Service has had its troubles during the first 25 years of its existence and the reorganization about to take place is unlikely to be free of them either. What must be acknowledged is Sir George's unceasing efforts to make the service a harmonious organization as much for the doctors who work it as for the patients in their care. More than most he deserves the gratitude of the medical profession for his labours on behalf of better medicine.

The burden of seeing the reorganization through will fall on Dr. Henry Yellowlees, who becomes C.M.O. after being with the Department since 1963. He brings to it a special knowledge of a problem that has come to be seen as lying at the heart of good medicine, and that is professional training with its ever-spreading ripples from undergraduate to postgraduate to continuing education. The profession's good wishes will certainly attend his accession to the post. 